\title{
Regulation of organ straightening and plant posture by an actin-myosin XI cytoskeleton
}

\section{$\operatorname{AUTHOR}(\mathrm{S}):$}

Okamoto, Keishi; Ueda, Haruko; Shimada, Tomoo; Tamura, Kentaro; Kato, Takehide; Tasaka, Masao; Morita, Miyo Terao; Hara-Nishimura, Ikuko

\section{CITATION:}

Okamoto, Keishi ...[et al]. Regulation of organ straightening and plant posture by an actin-myosin XI cytoskeleton. Nature Plants 2015, 1(4): 15031.

\section{ISSUE DATE:}

2015-03-23

URL:

http://hdl.handle.net/2433/197219

\section{RIGHT:}

(C) 2015 Macmillan Publishers Limited.; 許諾条件により本文ファイルは 2015-09-24に公開.; この論文は出版社版でありません。引用の際には 出版社版をご確認ご利用ください。; This is not the published version. Please cite only the published version. 


\title{
Regulation of organ straightening and plant posture by an actin- myosin XI cytoskeleton
}

\author{
Keishi Okamoto ${ }^{1,4}$, Haruko Ueda ${ }^{1,4}$, Tomoo Shimada ${ }^{1}$, Kentaro Tamura ${ }^{1}$, Takehide Kato ${ }^{2}$, \\ Masao Tasaka ${ }^{2}$, Miyo Terao Morita ${ }^{3}, \&$ Ikuko Hara-Nishimura $^{1} *$
}

${ }^{1}$ Graduate School of Science, Kyoto University, Kyoto 606-8502, Japan.

${ }^{2}$ Graduate School of Biological Sciences, Nara Institute of Science and Technology, Ikoma 630-0101, Japan.

${ }^{3}$ Graduate School of Bioagricultural Sciences, Nagoya University, Nagoya 464-8601, Japan.

${ }^{4}$ These authors contributed equally to this work.
${ }^{*}$ Correspondence should be addressed to I. H.-N. (e-mail: ihnishi@gr.bot.kyoto-u.ac.jp)

Plants are able to bend nearly every organ in response to environmental stimuli such as gravity and light ${ }^{1,2}$. After this first phase, the responses to stimuli are restrained by an independent mechanism, or even reversed, so that the organ will stop bending and attain its desired posture. This phenomenon of organ straightening has been called autotropism $^{3}$ and autostraightening ${ }^{4}$ and modelled as proprioception ${ }^{5}$. However, the machinery that drives organ straightening and where it occurs are mostly unknown. Here, we show that the straightening of inflorescence stems is regulated by an actinmyosin XI cytoskeleton in specialized immature fibre cells that are parallel to the stem and encircle it in a thin band. Arabidopsis mutants defective in myosin XI (specifically XIf and XIk) or ACTIN8 exhibit hyperbending of stems in response to gravity, an effect independent of the physical properties of the shoots. The actin-myosin XI cytoskeleton enables organs to attain their new position more rapidly than would an oscillating series of diminishing overshoots in environmental stimuli. We propose that the long actin filaments in elongating fibre cells act as a bending tensile sensor to perceive the organ's posture and trigger the straightening system. 
Myosin motor proteins occur in many classes, one of which is the plant-specific myosins XI that are involved in the bulk flow of cytoplasm (cytoplasmic streaming) in land plants and algae ${ }^{6,7}$. The Arabidopsis thaliana myosin XI gene family has 13 members, some of which are known to play roles in the movement of organelles including nuclei ${ }^{8-12}$. However, the cellular function of myosin XIf is unknown. We found that the lack of two myosin XI members (XIf and XIk) resulted in a kinked gross morphology (Supplementary Fig. 1a-c). Mature inflorescence stems and pedicels failed to grow straight in the null mutants myosin xif xik and myosin xil xi2 xib xif xik (Fig. 1a), but not in a quadruple mutant (myosin xil xi2 xib $x i k$ ) or in single mutants (myosin xif and myosin xik) (Fig. 1a and Supplementary Fig. 1c), possibly due to the redundant functions of myosins XIf and XIk. The kinked gross morphology of myosin xif xik was rescued by expressing each gene driven by its own promoter (Supplementary Fig. 1d). Among the 13 members, myosins XIf and XIk were expressed strongly at the transcript levels in second internodes of inflorescence stems and pedicels (Fig. 1b, Supplementary Fig. 2). These results imply that myosins XIf and XIk in the elongating and elongated organs are involved in adjusting plant posture.

We examined movements of various organs of these null mutants in response to gravity and light stimuli. To investigate the gravitropic response of growing inflorescence stems, the plants were placed horizontally. In the initial response phase, the stems of both the wild type and myosin xif-1 xik-2 bent $90^{\circ}$ to become vertical over a period of $80 \mathrm{~min}$ (Fig. 1c). Subsequently, the wild type continued to grow vertically, whereas myosin xif-1 xik-2 first overshot in one direction and then overshot in the other (Fig. 1c, $d$ and Supplementary Fig. 3a and Movie 1). In myosin xif-1 xik-2 and the double mutant alleles during gravitropism, bending was also enhanced in other elongating organs, including hypocotyls (Fig. 1e and Supplementary Fig. 3b), seedling roots (Fig. 1f and Supplementary Fig. 3c), and petioles (Supplementary Fig. 3d). The single mutants myosin xif and myosin xik showed no enhanced organ bending (Fig. 1c and Supplementary Fig. 3b, c). Furthermore, light enhanced the bending of myosin xif-1 xik-2 hypocotyls (Fig. 1g) and petioles (Fig. 1h). These results indicate that a combination of myosins XIf and XIk functions in stopping the bending of various organs in response to gravity or light. During gravitropism and phototropism of the organs, significant differences between the wild type and myosin xif-1 xik-2 were observed 
after a 2-h exposure to each stimulus (Fig. 1c, e-g), suggesting that $\sim 2 \mathrm{~h}$ is the time needed to detect the stopping of organ bending.

Gravitropism and phototropism have been proposed to consist of two separate processes: an organ-bending process and a straightening process to restrain the bending ${ }^{3-5}$. To investigate the straightening process, we conducted a clinostat test as shown in Fig. 2a (see Supplementary Fig. 4a for details): We first placed the plants horizontally for the minimum time needed to initiate gravitropic organ bending $(45 \mathrm{~min})$ and then rotated the plants on a horizontal clinostat at $5 \mathrm{rpm}$ in the dark to neutralize earth's unilateral gravitational pull. On the clinostat, in the first hour of rotation, the wild-type stems first grew with bending as a result of the preceding gravistimulation, and then the bending was restrained (Supplementary Fig. $4 \mathrm{~b}$ and Movie 2). The stems had straightened by $3 \mathrm{~h}$ of clinorotation (Fig. $2 \mathrm{~b}$ and Supplementary Movie 2). These results demonstrate that plants possess a straightening mechanism to stop organ bending and that the mechanism is triggered by organ bending.

To analyse the degree of straightening in each genetic background, we measured the angles of stem bending during the clinorotation treatment (Fig. 2a and Supplementary Fig. 4a). In most of the wild-type plants, the angle of stem bending decreased during 1 to $3 \mathrm{~h}$ of clinorotation (Fig. 2e), indicating that the stem was straightening. In contrast, in most of the myosin xif-1 xik-2, the stems continued to bend and coil (Fig. 2d and Supplementary Fig. 4c and Movie 2) and the angles of stem bending remarkably increased during 1 to $3 \mathrm{~h}$ of clinorotation (Fig. 2e and Supplementary Fig. 4d). The single mutant myosin xik showed mild defects in stem straightening on the clinostat, although myosin xif showed no defects (Fig. 2c, e and Supplementary Fig. 4d and Movie 2). In each genotype, the behavior of bending and straightening was not affected by stem lengths (Fig. 2f). These results indicate that myosin xif xik impaired the straightening process during the courses of gravitropism and phototropism. Accordingly, myosin xif xik is the first mutant to reveal authentic gravitropic and phototropic responses; that is, it is the first mutant that had been shown to respond to stimuli without the confounding effects of a straightening system.

The preceding results show that a combination of myosins XIf and XIk in the elongating and elongated organs regulates the straightening of organs and the adjustment of plant posture. Expression of a myosin XIk-GFP fusion protein under the control of the myosin XIf promoter (Supplementary Fig. 5a) significantly rescued the defect in straightening of 
inflorescence stems of myosin xik-2 and myosin xif-1 xik-2 (Fig. 3a) and kinked gross morphology of myosin xif-1 xik-2 (Supplementary Fig. 1d), suggesting that cells with an active myosin XIf-promoter have a key role in organ straightening to adjust plant posture. To determine which cells control straightening of the inflorescence stem, we examined the myosin XIf promoter activity in inflorescence stems of transgenic plants expressing $\beta$-glucuronidase (GUS) or fluorescent proteins under the control of the myosin XIf promoter. In a cross section of elongating stems, GUS signals driven by the myosin XIf promoter encircled the stem in a thin band (Fig. 3b). Higher magnification revealed that the band consisted of xylem fibre cells and interfascicular fibre cells (Fig. 3c, d). In a vertical section of elongating stems, GFP signals driven by the myosin XIf promoter were specifically detected in the fibre cell layer (Fig. 3e). On the other hand, YFP signals driven by the myosin $X I k$ promoter were detected in broader layers including the fibre cell layer than mCherry signals driven by the myosin XIf promoter (Fig. 3f and Supplementary Fig. 5b-d). These results suggest that straightening is controlled by immature fibre cells in elongating stems.

Mature fibre cells surrounded by thick secondary walls provide the inflorescence stem with mechanical strength. The physical features of both upper and basal parts of the stems (vacuolation degrees of the cells, vascular cell patterns, cell wall thickness, lignification, and hardness) of myosin xif-1 xik-2 are not significantly different from those of the wild-type (Supplementary Fig. 6). These results suggest that the enhanced bending phenotype of myosin xif-1 xik-2 is not caused by the physical features of the stems. On the other hand, the basal parts of the mutant stems hardly bent compared with those of the wild-type stems as a result of impairment of differential growth of cells on either side of the stem (Fig. 1d). Differential growth during the course of gravitropism and phototropism is caused by an asymmetrical auxin distribution ${ }^{13}$. Importantly, however, straightening is independent of an asymmetrical auxin distribution $^{14}$.

Because myosin XI proteins function in the organisation and dynamics of actin filaments in A. thaliana ${ }^{8,9,15}$ and the moss Physcomitrella patens ${ }^{16}$, we examined the actin-cytoskeleton by using transgenic plants expressing an actin fluorescent marker (Lifeact-Venus) under the control of the myosin XIf promoter. The fibre cells developed extremely long bundles of actin filaments along the longitudinal axis of the cells (Fig. 4a). Consistently, the fibre cells displayed vigorous cytoplasmic streaming with a maximal 
velocity of $\sim 8.5 \mu \mathrm{m} \mathrm{s}^{-1}$ (Fig. $4 \mathrm{~b}$ and Supplementary Movie 3 ). The cytoplasmic streaming was remarkably impaired in myosin xif xik and slightly impaired in myosin xif (Fig. 4b and Supplementary Movie 3), which confirms that myosin XIf is expressed in the fibre cells. The stem of the heterozygous mutant actin8 (frizzyl/+, fizl/+) showed abnormal phenotypes: enhanced bending in response to gravity (Fig. 4c), an inability to straighten on a clinostat (Fig. 4d and Supplementary Fig. 4e), and slower velocity of cytoplasmic streaming in fibre cells (Fig. 4e and Supplementary Movie 4). All of these phenotypes are very similar to those of myosin xif-1 xik-2, indicating that actin filaments together with myosins XIf and XIk are required for organ straightening. In addition, phenotypes similar to the kinked gross morphology of myosin xif-1 xik-2 were reported in various actin mutants including an actin2 actin 7 null mutant ${ }^{17}$, a semi-dominant mutant of ACTIN $2^{18}$, and a semi-dominant mutant of ACTIN8 $(f i z l)^{19}$. These results indicate that the actin-myosin XI cytoskeleton plays a role in adjusting plant posture through organ straightening.

The actin cytoskeleton has been shown to participate in gravity sensing by repositioning statolith amyloplasts ${ }^{20,21}$. However, no myosin XIf promoter activities were detected in endodermal cells of the stem (Figs. 3c and $4 \mathrm{a}$ and Supplementary Movie 5) or columella cells of root tips (Supplementary Fig. 7c), which are gravity-sensing cells of the stem ${ }^{22,23}$ and roots $^{24}$, respectively. Hence, organ straightening is independent of gravity sensing. On the other hand, myosin XIf promoter activities were detected in the vascular cells of hypocotyls and roots (Supplementary Fig. 7). Longitudinal actin cables have also been detected in root stele cells in maize ${ }^{25}$. These results suggest that the vascular cells in the stele are important for straightening of these organs. The actin inhibitor latrunculin B was found to induce phenotypes similar to those observed in the hypocotyls and roots of myosin xif-1 xik-2 (Fig. 1e, f): it enhanced bending in response to gravity in hypocotyls ${ }^{26,27}$ and $\operatorname{roots}^{28}$ of $A$. thaliana and it impaired straightening on a clinostat in the roots of maize, Medicago, and flax ${ }^{25}$. Furthermore, roots of an actin 2 mutant of $A$. thaliana growing in a microgravity environment exhibited hyper-skewing ${ }^{29}$. These phenotypes might be due to a defect of the actin-cytoskeleton-dependent straightening.

Our results provide a conceptual mechanistic advance in the understanding of gravitropism and phototropism: elongating immature fibre cells of the stem are key players in the straightening, in which an actin-myosin XI cytoskeleton controls the mechanism that 
enables organs to attain their new position more rapidly than would a series of diminishing overshoots in response to a tropic stimulus. How does the actin-myosin cytoskeleton function in straightening? Elongating fibre cells attain lengths of $\sim 1 \mathrm{~mm}$, which are many times the length of other plant cells, so that organ bending can lead to bending of the fibre cells. The fibre cells have longitudinally extra-long actin cables (Fig. 4a). Thus, we hypothesize that the long actin cables act as a sensor of fibre cell bending, which places the actin cable under tension. Actin cables under tension might activate mechanosensitive channels such as MCA1/2 $2^{30,31}$ to trigger the straightening system as actin stress fibres can activate mechanosensitive channels in animal cells ${ }^{32}$. Considering the previous report ${ }^{8}$, myosin xif xik might exhibit a defect in the transvacuolar cytoplasmic strand formed by the actin cables, resulting in the impairment of vacuolar function in the mutant cells. Hence, one cannot exclude a possibility of an involvement of vacuoles in straightening.

\section{METHODS}

Plant materials and growth conditions. Arabidopsis thaliana L. (Heynh) accession Columbia-0 (CS60000) was used as the wild type. We established the T-DNA insertion lines myosin xif-1 (SALK_094787) and myosin xif-2 (SAIL_514_H11) in this study. The T-DNA insertions were confirmed by PCR with primers designed using iSECT tools (Supplementary Fig. 1a). Other lines have been described previously, namely: myosin xib-1 (SALK_113062) ${ }^{33}$, myosin xig-1 (SALK_018032) ${ }^{33}$, myosin xik-1 (SALK_136682) ${ }^{8}$, myosin xik-2 (SALK_ 067972) $)^{8}$, myosin xik-3 (SALK_018764) ${ }^{8}$, myosin xik-4 (SALK_152496) ${ }^{8}$, myosin xil-2 $(\text { SALK_022140 })^{8}$, myosin xi2-2 (SALK_ 055785) ${ }^{8}$, and $f i z 1^{19}$. Seeds of all lines except for fizl and a binary vector for plasma membrane marker (pm-rb) ${ }^{34}$ were obtained from the Arabidopsis Biological Resource Center. Plants were initially grown on plates of MurashigeSkoog medium containing $1 \%(\mathrm{w} / \mathrm{v})$ sucrose, $0.5 \%(\mathrm{w} / \mathrm{v}) \mathrm{MES}-\mathrm{KOH}(\mathrm{pH} 5.7)$, and $0.5 \%$ (w/v) Gellan gum (Wako), under continuous light at $22{ }^{\circ} \mathrm{C}$ for 2 to 3 weeks before being transferred to soil. For the analyses of tropic responses of hypocotyls and roots, seedlings were grown vertically along the surface of the medium for 3 days in the dark.

Gravitropism assay of inflorescence stems. Intact plants with 4 to $8 \mathrm{~cm}$ primary stems were placed horizontally in nondirectional dim light $\left(1 \mu \mathrm{mol} \mathrm{m} \mathrm{m}^{-2}\right)$. Photographs were taken automatically every $10 \mathrm{~min}$ by digital camera (Canon). Stem curvature was defined as the 
angle formed between the growing direction of the apex and the horizontal base line and was measured on the digital images using Image J software (http://rsb.info.nih.gov/ij/).

Gravitropism assay of petioles, hypocotyls and roots. For petioles, 14-day-old plants grown on agar plates were reoriented by $90^{\circ}$ and photographed after $24 \mathrm{~h}$ growth in darkness. For hypocotyls and roots, 3-day-old etiolated seedlings grown on 7 sets of agar plates were reoriented by $90^{\circ}$ in darkness. A set of the agar plates was moved to light condition and photographed at every time point $(0,1.5,3,6,9,16$ and $24 \mathrm{~h})$. The angle was measured between a horizontal base line and either the root tip or the apical-most segment of the hypocotyl before the hook.

Phototropic assay of petioles and hypocotyls. For petioles, 14-day-old plants grown on agar plates were irradiated for $24 \mathrm{~h}$ with unilateral blue light from an LED array at $0.01 \mu \mathrm{mol} \mathrm{m}{ }^{-2}$ $\sec ^{-1}$. For hypocotyls, 3-day-old etiolated seedlings grown on agar plates were irradiated for 4 $\mathrm{h}$ with unilateral blue light from an LED array at $0.1 \mu \mathrm{mol} \mathrm{m} \mathrm{mec}^{-1}$. Seedlings were photographed either under blue light and curvature of the hypocotyl was measured as described for the gravitropism assay.

Clinostat analysis of inflorescence stems. Plants with 4- to 8-cm-long primary stems were set on clinostat disc with a $7-\mathrm{cm}$ radius and were gravistimulated by placing horizontally. The minimum time needed to initiate gravitropic organ bending was determined to be $45 \mathrm{~min}$. After gravistimulation, the plants were rotated on a horizontal clinostat for $3-10 \mathrm{~h}$ as shown in Fig. 2a and Supplementary Fig. 4a. The angles of stem bending during 1 to $3 \mathrm{~h}$ of clinorotation were measured. All procedures were performed in the dark at $23^{\circ} \mathrm{C}$.

Expression analysis of myosin $X I$. To detect GUS activity, tissue was immersed in $90 \%$ ice-cold acetone for $15 \mathrm{~min}$ and then incubated in GUS staining solution (100 mM sodium phosphate $\mathrm{pH}$ 7.0, $10 \mathrm{mM}$ EDTA, $10 \mathrm{mM}$ ferricyanide, $10 \mathrm{mM}$ ferrocyanide, $0.1 \%$ Triton $\mathrm{X}-100,0.52 \mathrm{mg} \mathrm{mL}^{-1}$ 5-bromo-4-chloro-3-indoryl- $\beta$-D-glucuronic acid) overnight at $37^{\circ} \mathrm{C}$. After being cleared in 70\% ethanol, samples were observed with a light microscope. For semi-thin sections $(8 \mu \mathrm{m})$, GUS-stained inflorescence stems were embedded in Technovit 7100 (Heraeus Kulzer), sectioned on a rotary microtome (Leica Microsystems), and stained with $0.05 \%$ aqueous neutral red for $\sim 5 \mathrm{sec}$ to contrast the cell wall. For thick sections (100 $\mu \mathrm{m})$, GUS-stained inflorescence stems were embedded in 5\% agar and sectioned with a vibrating blade microtome (Leica Microsystems).

Vertical sections of the inflorescence stem were prepared as described previously ${ }^{35}$. Briefly, double-sided adhesive tape was cut and stuck onto a glass slide. A four-week-old, 
inflorescence stem segment (less than $1 \mathrm{~cm}$ long) was excised from the region 1 to $2 \mathrm{~cm}$ below the apex of the stem and stuck to the tape. The segment was cut longitudinally using a razor blade. Fluorescent images were obtained with a confocal laser-scanning microscope (LSM780, Zeiss) using a water-immersion objective $(63 \times 1.20 \mathrm{NA})$ and dry objectives $(20 \times$ $0.80 \mathrm{NA}$ and $5 \times 0.25 \mathrm{NA}$ ). The laser wavelengths used include $488 \mathrm{~nm}$ (GFP and YFP) and $543 \mathrm{~nm}$ (mCherry). The images were analysed using LSM image software (Carl Zeiss), and were processed using ImageJ (NIH) and Photoshop (Adobe Systems) software.

Observation of cytoplasmic streaming in fibre cells. To examine cytoplasmic streaming in fibre cells, the vertical sections were observed in bright field using a light microscopy (LSM 510 META, Zeiss) with a $63 \times 1.2$ numerical aperture water-immersion objective. The interfascicular fibre cells are anatomically distinguishable from other cells based on their taper ends and being more than five times longer than their neighboring parenchyma cells or endodermal cells ${ }^{36}$. The differential interference contrast images were taken at 1-sec intervals for $1 \mathrm{~min}$. For estimation of velocity, moving plastids in 4 or 5 randomly-selected sequential-frames of 12-15 time-lapse images $(143 \times 143 \mu \mathrm{m})$ were tracked manually using ImageJ software.

Online Content Methods, along with any additional Extended Data display items and Source Data, are available in the online version of the paper; references unique to these sections appear only in the online paper.

1 Darwin, C. The Power of Movements in Plants. (D. Appleton and Company, 1880).

2 Gilroy, S. \& Masson, P. H. Plant Tropisms. (Blackwell, 2008).

3 Stankovic, B., Volkmann, D. \& Sack, F. D. Autotropism, automorphogenesis, and gravity. Physiol. Plant. 102, 328-335 (1998).

4 Iino, M. Toward understanding the ecological functions of tropisms: interactions among and effects of light on tropisms. Curr. Opin. Plant Biol. 9, 89-93 (2006).

5 Bastien, R., Bohr, T., Moulia, B. \& Douady, S. Unifying model of shoot gravitropism reveals proprioception as a central feature of posture control in plants. Proc. Natl. Acad. Sci. USA 110, 755-760 (2013).

6 Shimmen, T. \& Yokota, E. Cytoplasmic streaming in plants. Curr. Opin. Cell Biol. 16, 68-72 (2004).

7 Tominaga, M. \& Nakano, A. Plant-specific myosin XI, a molecular perspective. Front. Plant Sci. 3, 211 (2012).

8 Ueda, H. et al. Myosin-dependent endoplasmic reticulum motility and F-actin organization in plant cells. Proc. Natl. Acad. Sci. USA 107, 6894-6899 (2010).

9 Peremyslov, V. V., Prokhnevsky, A. I. \& Dolja, V. V. Class XI myosins are required for 
development, cell expansion, and F-Actin organization in Arabidopsis. Plant Cell 22, 1883-1897 (2010).

10 Sparkes, I. A. Motoring around the plant cell: insights from plant myosins. Biochem. Soc. Trans. 38, 833-838 (2010).

11 Avisar, D., Abu-Abied, M., Belausov, E. \& Sadot, E. Myosin XIK is a major player in cytoplasm dynamics and is regulated by two amino acids in its tail. J. Exp. Bot. 63, 241-249 (2012).

12 Tamura, K. et al. Myosin XI-i links the nuclear membrane to the cytoskeleton to control nuclear movement and shape in Arabidopsis. Curr. Biol. 23, 1776-1781 (2013).

13 Esmon, C. A. et al. A gradient of auxin and auxin-dependent transcription precedes tropic growth responses. Proc Natl Acad Sci U S A 103, 236-241 (2006).

14 Haga, K. \& Iino, M. Asymmetric distribution of auxin correlates with gravitropism and phototropism but not with autostraightening (autotropism) in pea epicotyls. J Exp Bot 57, 837-847 (2006).

15 Cai, C., Henty-Ridilla, J. L., Szymanski, D. B. \& Staiger, C. J. Arabidopsis Myosin XI: A Motor Rules the Tracks. Plant Physiol (2014).

16 Vidali, L. et al. Myosin XI is essential for tip growth in Physcomitrella patens. Plant Cell 22, 1868-1882 (2010).

17 Kandasamy, M. K., McKinney, E. C. \& Meagher, R. B. A single vegetative actin isovariant overexpressed under the control of multiple regulatory sequences is sufficient for normal Arabidopsis development. Plant Cell 21, 701-718 (2009).

18 Lanza, M. et al. Role of actin cytoskeleton in brassinosteroid signaling and in its integration with the auxin response in plants. Dev. Cell 22, 1275-1285 (2012).

19 Kato, T., Morita, M. T. \& Tasaka, M. Defects in dynamics and functions of actin filament in Arabidopsis caused by the dominant-negative actin fizl-induced fragmentation of actin filament. Plant Cell Physiol. 51, 333-338 (2010).

20 Nakamura, M., Toyota, M., Tasaka, M. \& Morita, M. T. An Arabidopsis E3 ligase, SHOOT GRAVITROPISM9, modulates the interaction between statoliths and F-actin in gravity sensing. Plant Cell 23, 1830-1848 (2011).

21 Blancaflor, E. B. Regulation of plant gravity sensing and signaling by the actin cytoskeleton. Am J Bot 100, 143-152 (2013).

22 Fukaki, H. et al. Genetic evidence that the endodermis is essential for shoot gravitropism in Arabidopsis thaliana. Plant J. 14, 425-430 (1998).

23 Hashiguchi, Y., Tasaka, M. \& Morita, M. T. Mechanism of higher plant gravity sensing. Am. J. Bot. 100, 91-100 (2013).

24 Blancaflor, E. B., Fasano, J. M. \& Gilroy, S. Mapping the functional roles of cap cells in the response of Arabidopsis primary roots to gravity. Plant Physiol. 116, 213-222 (1998).

25 Hou, G., Mohamalawari, D. R. \& Blancaflor, E. B. Enhanced gravitropism of roots with a disrupted cap actin cytoskeleton. Plant Physiol 131, 1360-1373 (2003).

26 Yamamoto, K. \& Kiss, J. Z. Disruption of the actin cytoskeleton results in the promotion of gravitropism in inflorescence stems and hypocotyls of Arabidopsis. Plant Physiol. 128, 669-681 (2002).

27 Palmieri, M. \& Kiss, J. Z. Disruption of the F-actin cytoskeleton limits statolith 
movement in Arabidopsis hypocotyls. J Exp Bot 56, 2539-2550 (2005).

28 Hou, G. et al. The promotion of gravitropism in Arabidopsis roots upon actin disruption is coupled with the extended alkalinization of the columella cytoplasm and a persistent lateral auxin gradient. Plant J. 39, 113-125 (2004).

29 Nakashima, J., Liao, F., Sparks, J. A., Tang, Y. \& Blancaflor, E. B. The actin cytoskeleton is a suppressor of the endogenous skewing behaviour of Arabidopsis primary roots in microgravity. Plant Biol (Stuttg) 16 Suppl 1, 142-150 (2014).

30 Nakagawa, Y. et al. Arabidopsis plasma membrane protein crucial for $\mathrm{Ca} 2+$ influx and touch sensing in roots. Proc Natl Acad Sci U S A 104, 3639-3644 (2007).

31 Yamanaka, T. et al. MCA1 and MCA2 that mediate Ca2+ uptake have distinct and overlapping roles in Arabidopsis. Plant Physiol 152, 1284-1296 (2010).

32 Hayakawa, K., Tatsumi, H. \& Sokabe, M. Actin stress fibers transmit and focus force to activate mechanosensitive channels. J Cell Sci 121, 496-503 (2008).

33 Peremyslov, V. V., Prokhnevsky, A. I., Avisar, D. \& Dolja, V. V. Two class XI myosins function in organelle trafficking and root hair development in Arabidopsis. Plant Physiol. 146, 1109-1116 (2008).

34 Nelson, B. K., Cai, X. \& Nebenfuhr, A. A multicolored set of in vivo organelle markers for co-localization studies in Arabidopsis and other plants. Plant J 51, 1126-1136 (2007).

35 Saito, C., Morita, M. T., Kato, T. \& Tasaka, M. Amyloplasts and vacuolar membrane dynamics in the living graviperceptive cell of the Arabidopsis inflorescence stem. Plant Cell 17, 548-558 (2005).

36 Ye, Z. H., Freshour, G., Hahn, M. G., Burk, D. H. \& Zhong, R. Vascular development in Arabidopsis. Int. Rev. Cytol. 220, 225-256 (2002).

Supplementary Information is available in the online version of the paper.

Acknowledgements We are grateful to Tobias Baskin (University of Massachusetts), Alistair M. Hetherington (University of Bristol), and James Raymond (Eigoken) for critical readings of this manuscript and to Moritoshi Iino (Osaka City University), Tomomi Suzuki (Kyoto University), Akira Nagatani (Kyoto University) for helpful discussion. We are also grateful to Shoko Hongo (Tohoku University), Kazuhiko Nishitani (Tohoku University), Masatsugu Toyota (NAIST) and Masatoshi Taniguchi (NAIST) for their technical support; to Valerian V. Dolja (Oregon State University) for his donation of the ProXIk:XIk-YFP construct, transgenic seeds, and anti-XIk antibody; to Takashi Ueda (University of Tokyo) for his donation of the Pro35S:Lifeact-Venus construct; to Tsuyoshi Nakagawa (Shimane University) for his donation of Gateway vectors; and to the ABRC for providing seeds of $A$. thaliana T-DNA insertion mutants. This work was supported by Specially Promoted Research of Grant-in-Aid for Scientific Research to I.H-.N. (no. 22000014), Grants-in-Aid for Scientific Research to 
K.O. (no. 23.2) and to H.U. (nos. 21200065 and 25440132) from the Japan Society for the Promotion of Science (JSPS).

Author Contributions K.O., H.U., T.S. and I.H-N conceived the study; K.O. and H.U. designed the experiments; K.O. generated myosin mutants and analysed tropic responses. K.O. and H.U. generated transgenic plants and analysed straightening; K.O., T.K., M.T. and M.T.M. analysed gravitropic responses; K.O., H.U., T.S., K.T. and I.H-N participated in discussion; K.O., H.U., T.S. and I.H-N wrote the manuscript.

Author Information The authors declare no competing financial interests. 


\section{FIGURE LEGENDS}

\section{Figure 1 | Deficiency of myosins XIf and XIk enhances organ bending in response to} gravity and light stimuli. a, Upper inflorescence stems of plants grown upright. b, Transcript levels of myosin XI in 2nd internodes (orange) and vegetative rosette leaves (grey). Data were obtained from Public Arabidopsis eFP Browser. c, Kinetics of gravitropism of inflorescence stems after placing the plant horizontally. Error bars indicate SE. * $P<0.005 ; * *, P<0.001$ (Student's $t$ test). d, Time course of gravitropism of inflorescence stem. See also Supplementary Movie 1. e, Kinetics of gravitropism of hypocotyls. At time 0, etiolated seedlings were placed horizontally. ${ }^{*}, P<0.001 ; * *, P<1.0 \times 10^{-8}$ (Student's $t$ test). f, Kinetics of gravitropism of roots. Roots bend in opposite direction to that of hypocotyls. *, $P$ $<0.001 ; * *, P<1.0 \times 10^{-8}$ (Student's $t$ test). g, Kinetics of phototropism of hypocotyls. Etiolated seedlings were irradiated with unilateral, continuous blue light. ${ }^{*}, P<0.05$; ${ }^{* *}, P<$ 0.005 (Student's $t$ test). Error bars in e-g indicate SD. h, Phototropism of petioles. Plants were irradiated with continuous unilateral blue light (indicated by blue arrow). Each number indicates the same leaf. See Supplementary Table 1 for statistical analysis of kinetics in $\mathbf{c}$ and e-g. Bars $=1 \mathrm{~cm}$.

\section{Figure 2 | Myosins XIf and XIk are required for straightening of inflorescence stems.}

Clinostat test for straightening in the absence of a consistent gravity signal. a, Schematic depiction of clinostat test. After horizontal placement for $45 \mathrm{~min}$, plants were rotated at $5 \mathrm{rpm}$ for 3-10 h on a horizontal clinostat; $\boldsymbol{g}$ indicates the direction of gravity. See Supplementary Fig. 4a for more detail. b-d, Representative images of wild type, myosin xik-2, and myosin xif-1 xik-2 during clinorotation. See also Supplementary Fig. 4b, c and Movie 2. e, The angles of stem bending were measured as indicated in a. al and a 3 indicate angles at $1 \mathrm{~h}$ and $3 \mathrm{~h}$, respectively. According to the angles, frequency of plants showing straightening (light blue) or bending (dark blue) was determined. Numbers on bars indicate number of plants. f, Frequency of plants showing straightening (light blue) or bending (dark blue) are shown for plants with the indicated primary stem lengths. 


\section{Figure 3 | Fibre cells expressing myosins XIf and XIk play a role in straightening. a,}

Clinostat test for straightening of wild type (left), myosin xik-2 (middle), and myosin xif-1 xik-2 (right), all of which express ProXIf:XIk-GFP, as in Fig. 2. b, c, GUS staining of inflorescence stem of plant expressing ProXIf:GUS. Image of 100- $\mu \mathrm{m}$ section (b). Image of $8-\mu \mathrm{m}$ section stained with neutral red (c). IF, interfascicular fibre cells; XF, xylem fibre cells; xy, xylem; ph, phloem. Bars $=100 \mu \mathrm{m}(\mathbf{b})$ and $50 \mu \mathrm{m}(\mathbf{c}) . \mathbf{d}$, Schematic depiction of inflorescence stem. Left, vertical section; right, cross section. e, f, Vertical sections of inflorescence stem of a transgenic plant expressing either ProXIf:XIk-GFP in myosin xif-1 xik-2 (e) or both ProXIf:mCherry-ER and ProXIk:XIk-YFP in wild type (f). Asterisks indicate autofluorescence in cortex layers.

\section{Figure 4 | Fibre cells develop extremely long actin cables and a defect in ACTIN8 causes} the abnormal phenotypes similar to those of myosin xif xik. a, Vertical section of primary inflorescence stem (8 cm height) of a transgenic plant expressing Pro35S:mCherry-PM (plasma membrane marker) and ProXIf:Lifeact-VENUS (actin marker). Maximum intensity projections reconstituted from 14 sequential images taken along the optical z-axis at $0.5-\mu \mathrm{m}$ intervals (left column) and single images (right column). See Supplementary Movie 5 for the three-dimensional organisation. b, Maximal velocity of plastids in fibre cells. *, $P<0.005$; **, $P<1.0 \times 10^{-25}$ (Student's $t$ test). c, Kinetics of gravitropism of actin8 $(f i z l /+)$ inflorescence stem. ${ }^{* *}, P<5.0 \times 10^{-5}$ (Student's $t$ test). d, Clinostat test for straightening of actin $8(f i z l /+)$. e, Maximum velocity of plastids in fibre cells of actin 8 (fizl/ + ). **, $P<1.9 \times 10^{-9}$ (Student's $t$ test). Error bars in $\mathbf{b}, \mathbf{c}$, and $\mathbf{e}$ indicate SE. See Supplementary Table 2 for statistical analysis of $\mathbf{b}, \mathbf{c}$, and $\mathbf{e}$. 
a

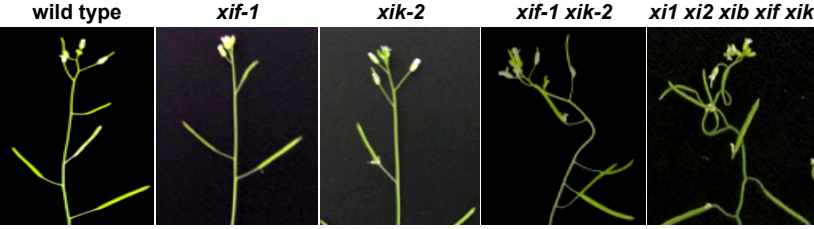

b

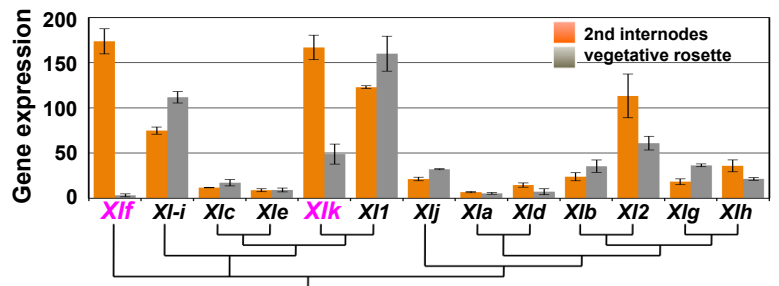

C Stem curvature (gravi-stimulation)
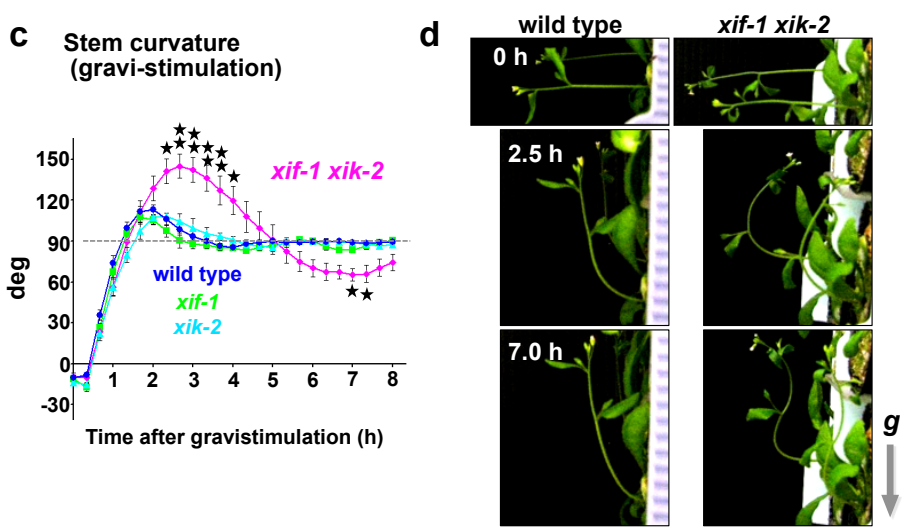

e $\begin{gathered}\text { Hypocotyl curvature } \\ \text { (gravi-stimulation) }\end{gathered}$

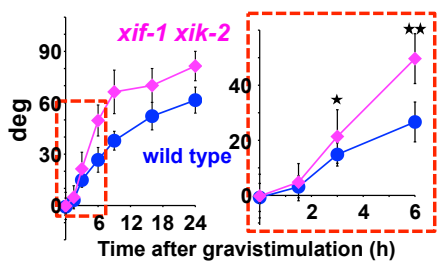

f Root curvature (gravi-stimulation)

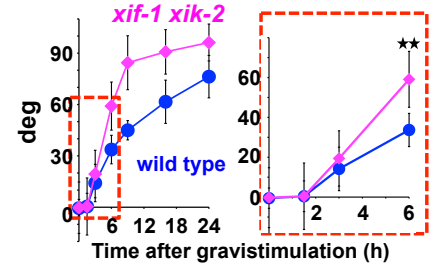

g Hypocotyl curvature

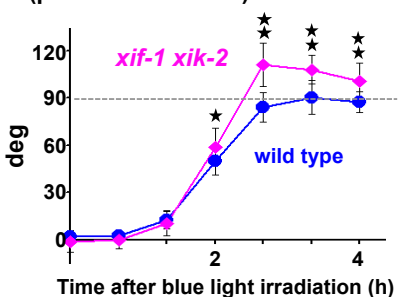

h

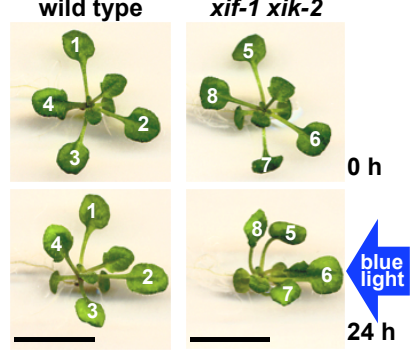


a

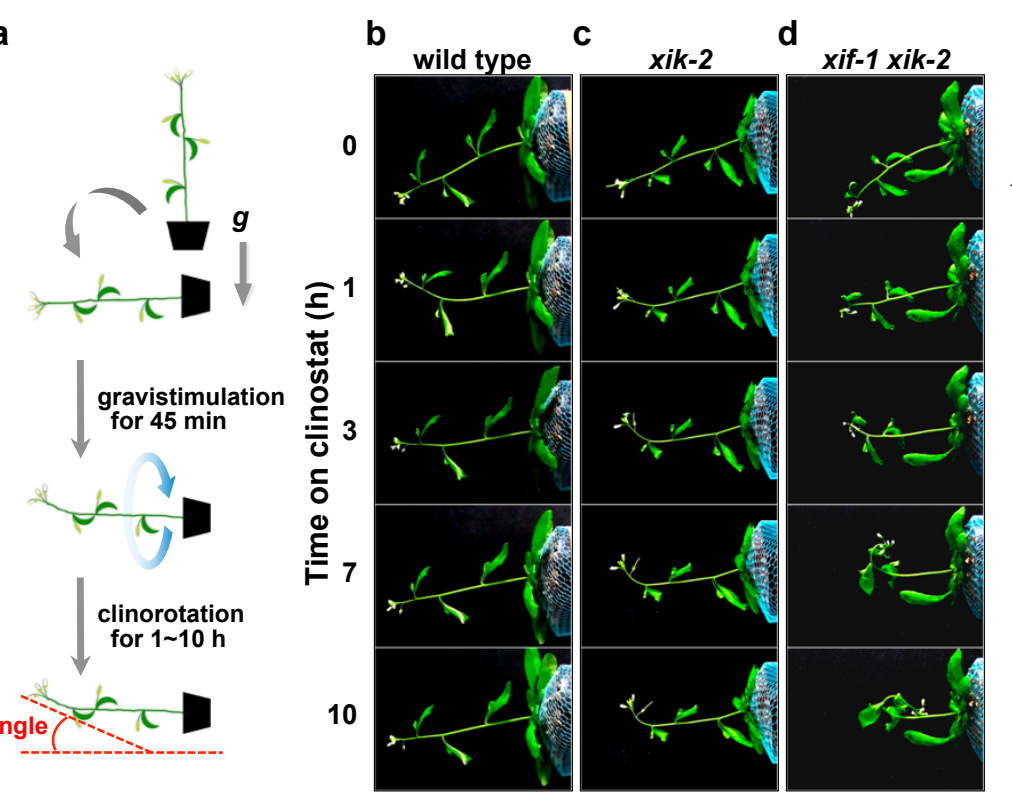

e

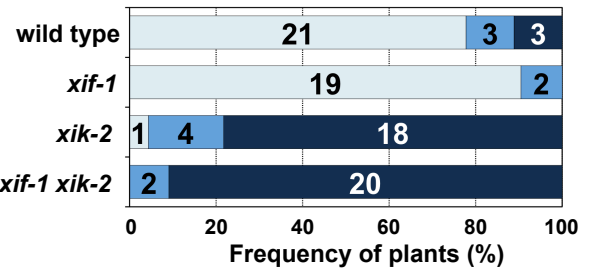

f wild type

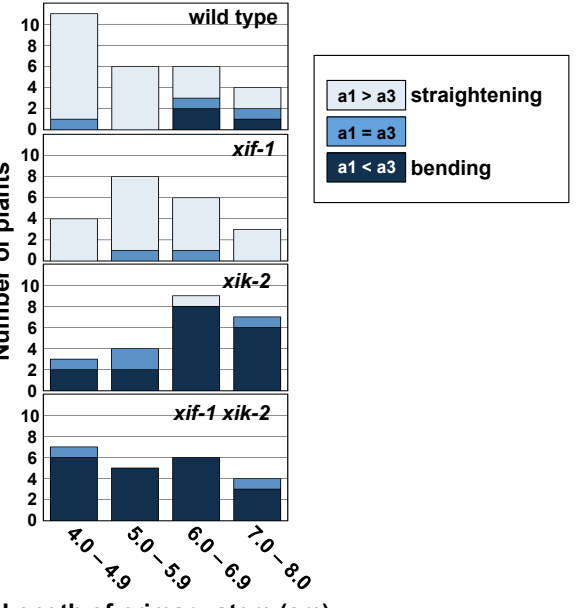

Length of primary stem $(\mathrm{cm})$ 

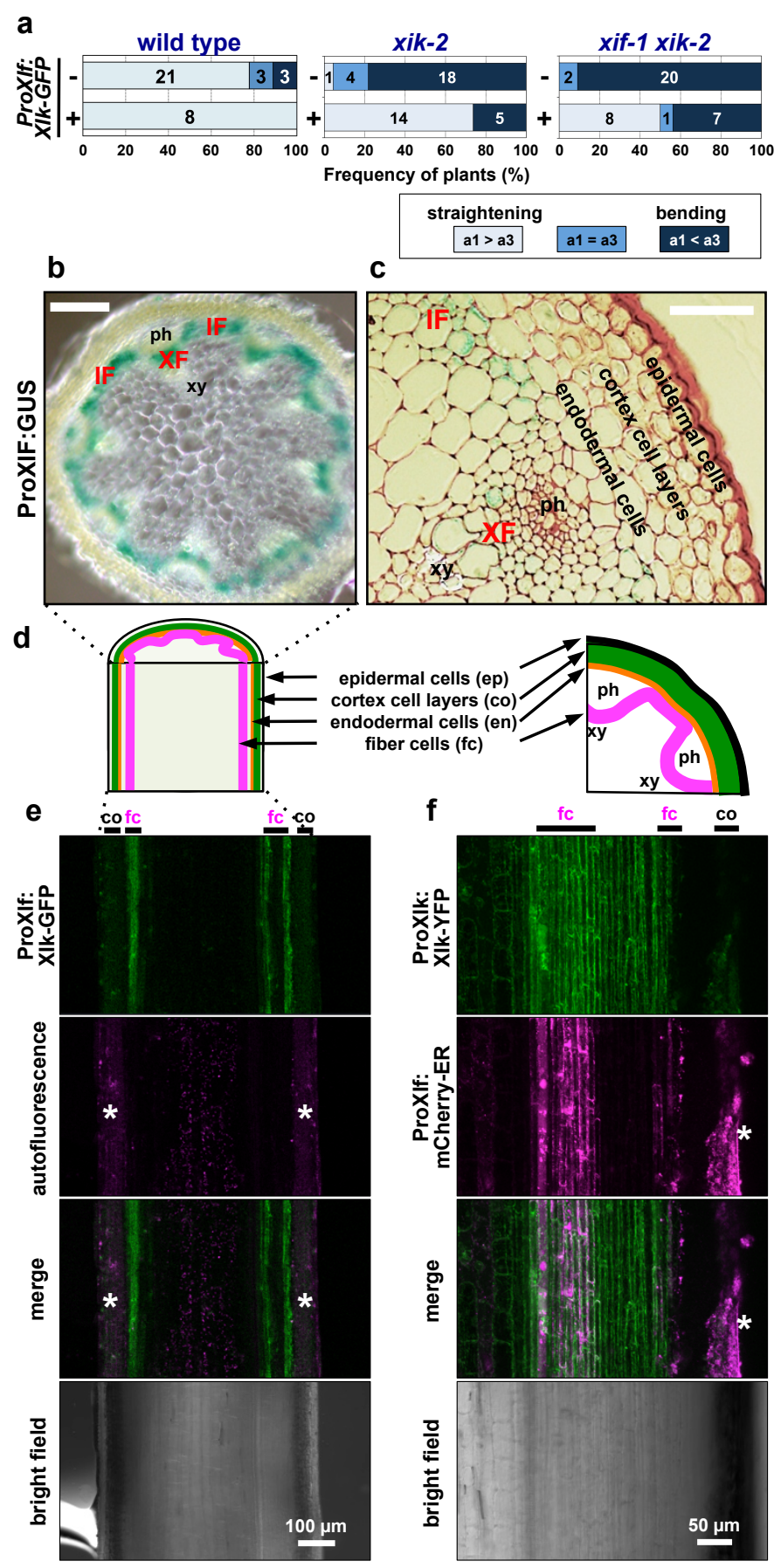
a

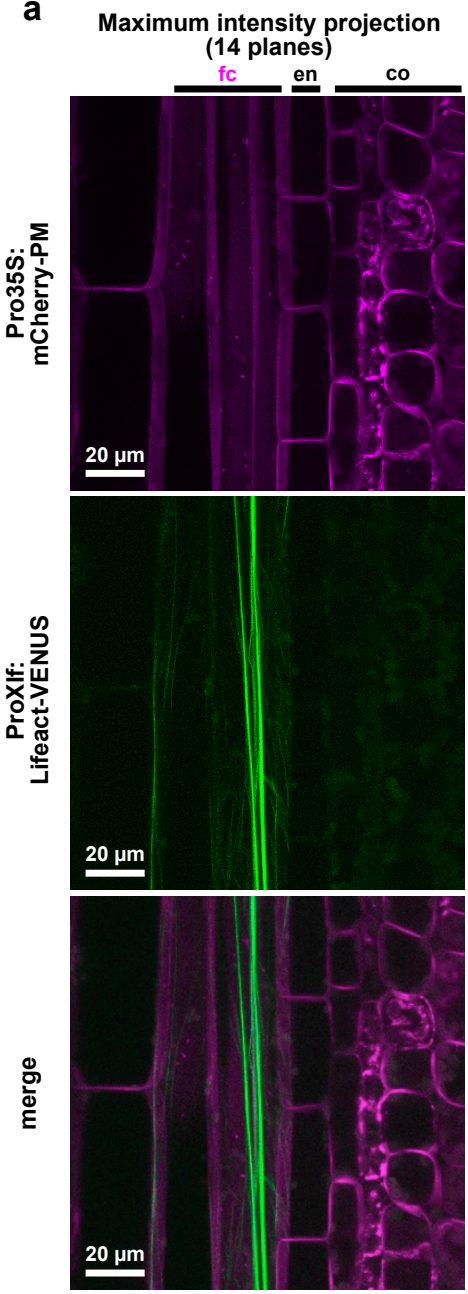

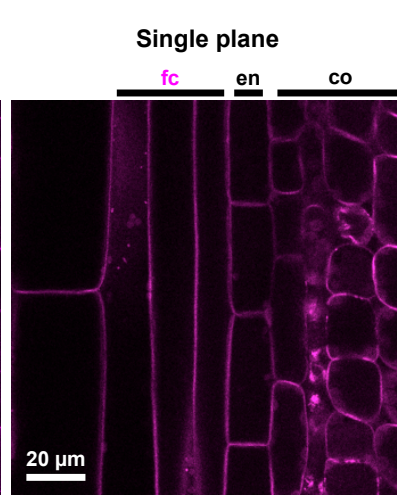
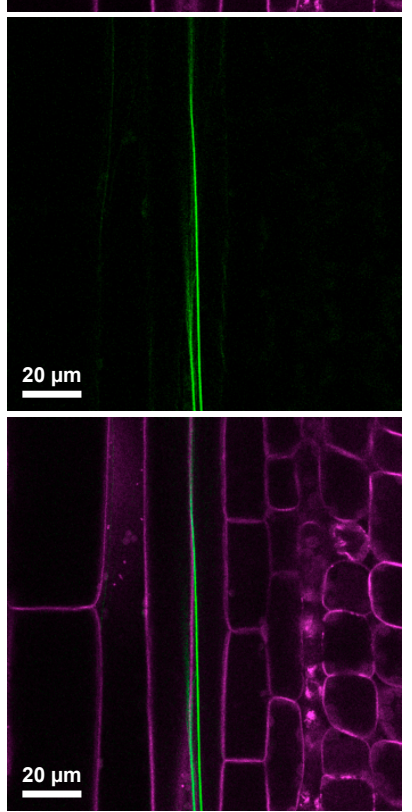

b

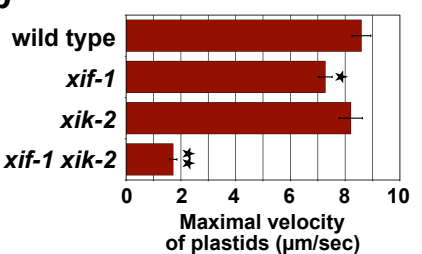

C

Stem curvature

(gravi-stimulation)

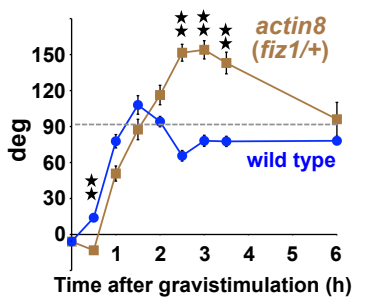

d

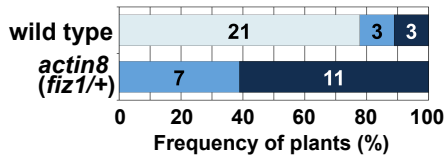

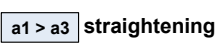

a1 $=\mathrm{a} 3$

a1 $<3$ bending

e

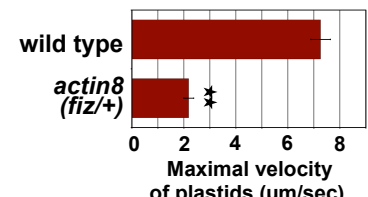

